\title{
Teorías implícitas sobre escritura académica y su enseñanza: contrastes entre el ingreso, la transición y el egreso universitarios*
}

\section{Implicit Theories about Academic Writing: Contrasts between University Entry, Transition and Graduation}

Recepción: 15 Mayo 2018 | Aceptación: 02 Agosto 2019

\author{
FEDERICO NaVARro ${ }^{\mathrm{a}}$ \\ Universidad de O'Higgins, Chile \\ ORCID: http://orcid.org/0000-0001-9131-3245 \\ Bárbara Mora-Aguirre \\ Universidad de Chile, Chile \\ ORCID: http://orcid.org/0000-0001-8023-4699
}

a Autor de correspondencia. Correo electrónico: navarro@uoh.cl

Para citar este artículo: Navarro, F., \& Mora-Aguirre, B. (2019). Teorías implícitas sobre escritura académica y su enseñanza: contrastes entre el ingreso, la transición y el egreso universitarios. Universitas Psychologica 18(3), 1-17. https://doi.org/10.11144/Javeriana.upsy1 8-3.tiea

\section{RESUMEN}

La educación superior incluye la enculturación de los estudiantes en las formas esperadas de comunicarse disciplinarmente, pero este proceso puede verse obstaculizado por ciertas teorías implícitas sobre la escritura (transcripción, talento, inspiración individual, habilidad básica, vocabulario y ortografía, objeto no enseñable) que difieren de su carácter de actividad retórica compleja, situada y epistémica. Esta investigación reconstruye y contrasta la presencia de estas teorías en tres etapas formativas (ingreso, transición y egreso), a partir de las respuestas de 8182 estudiantes universitarios. Los resultados, evaluados con prueba chi cuadrado de Pearson de bondad de ajuste, muestran que los estudiantes ingresan con ciertas teorías implícitas (escritura como ortografía y transcripción del pensamiento, aprendizaje mediante la práctica y a través del currículum y niveles formativos), pero no con otras (escritura como habilidad básica); además, la formación universitaria transforma parcialmente algunas (escritura como inspiración individual y transcripción del pensamiento), pero no otras (importancia relativa de la adecuación situacional y la estructura textual), e incorpora expectativas sobre tareas (posicionamiento propio en ensayos, uso de citas en explicaciones, contrastes en comparaciones). Comprender la distribución y los grados de estabilidad o transformación de teorías implícitas sobre la escritura no solo hace aportes al conocimiento científico, sino que también informa iniciativas de alfabetización académica, de escritura a través del currículum y de apoyo y orientación de los aprendizajes.

\section{Palabras clave}

teorías implícitas; representaciones; enseñanza de la escritura; escribir a través del currículum; educación superior.

\section{ABSTRACT}

Higher education includes students' enculturation into expected disciplinary communication, but this process might be hindered by certain implicit theories about writing (transcription; talent; individual inspiration; basic ability; vocabulary and mechanics; non-teachable object) that differ from its actual configuration as a complex, situated 
and epistemic rhetorical activity. This article retrieves and contrast these theories in three different educational stages (entry, transition, and graduation) based on answers from 8182 university students. After applying Pearson's Chisquare Goodness of Fit Test, results show that students enter university with certain implicit theories (writing as mechanics and transcription of thought; learning through practice and across the curriculum and educational stages), but not with others (writing as a basic ability); furthermore, higher education trajectories partially transform certain theories (writing as individual inspiration and transcription of thought), but do not affect others (situational adequacy and textual structure relative importance), while incorporating expectations about writing tasks (personal stance in essays; use of citations in explanations; contrasts in comparisons). Understanding the distribution and the degrees of stability or transformation of implicit theories about writing not only makes contributions to scientific knowledge, but it also informs literacy teaching, writing across the curriculum, and students' support programs and initiatives.

Keywords

implicit theories; representations; writing teaching; writing across the curriculum; higher education.

La sociedad del conocimiento presenta contextos comunicativos, interlocutores, propósitos y soportes tecnológicos cada vez más complejos, diversificados y fluctuantes. Por este motivo, la participación plena en sociedad requiere hoy en día escritoras y escritores avanzados que puedan comprender y ajustarse a distintas expectativas comunicativas, transferir conocimientos desde experiencias previas y aprender de manera continua nuevas formas de comunicar (Bazerman, 2013; Starke-Meyerring \& Paré, 2011).

La expansión de la educación superior como fenómeno global (Fernández Lamarra \& Costa de Paula, 2011) responde en parte a esta necesidad de formar escritores avanzados. El perfil de egreso de un graduado universitario no consiste solamente en la adquisición de los conocimientos especializados y las competencias requeridas en su carrera y en su futuro campo de inserción laboral, sino en una verdadera enculturación en las formas de hacer, de pensar y de comunicar de las culturas disciplinares (Prior \& Bilbro, 2011). La escritura académica en educación superior no es una competencia genérica, simple y 'blanda', adquirida en las etapas iniciales de alfabetización. Por el contrario, abarca un rico conjunto de prácticas letradas diversificadas tanto situacional como culturalmente que se corresponden con ciertas formas de pensar y de actuar en sociedad, y que suelen tipificarse en un conjunto de géneros discursivos característicos definidos por las expectativas, en permanente disputa y trasformación, que tienen sobre ellos los usuarios (Bazerman, 2012). Una graduada de la Licenciatura en Ciencias construirá conocimientos a partir del uso de datos empíricos recogidos mediante procedimientos replicables, y esta práctica se corresponderá con el informe de laboratorio como género discursivo y con ciertos patrones textuales, como la despersonalización del sujeto responsable del reporte de hallazgos. Por el contrario, una graduada de la Licenciatura en Letras Hispánicas construirá conocimientos a partir del uso creativo y crítico de fuentes y la exploración de la subjetividad en la observación y conceptualización de fenómenos, y esta práctica se corresponderá con el ensayo como género discursivo y la primera persona singular para destacar la perspectiva particular del autor. Además de esta variación disciplinar, los estudiantes universitarios escriben diferentes conjuntos de géneros discursivos para dirigirse a audiencias diversas y cumplir con propósitos distintos; los géneros discursivos esperados variarán según la etapa formativa, el grado de experticia y el rol de cada estudiante (Swales, 2004).

Esta ubicuidad, diversidad, riqueza y flexibilidad de las prácticas letradas en educación superior tiene como contracara su invisibilización curricular como objeto válido y necesario de enseñanza y aprendizaje (Schleppegrell, 2004). Los estudiantes por lo general no reciben instrucciones explícitas para realizar las tareas de escritura asignadas, o bien las instrucciones son poco precisas o se refieren a aspectos de formato y extensión. Sin embargo, estas tareas pueden resultar sumamente específicas, vinculadas a variables disciplinares e incluso particulares del curso y docente, y en muchos casos se corresponden con actividades y géneros discursivos que los 
estudiantes nunca han escrito antes (Carlino, 2013). Además, en general, tampoco se les brinda una retroalimentación vinculada a la elaboración textual ni oportunidades para revisar y reescribir sus textos, a pesar de que los escritores con mayor experticia son aquellos que incorporan el proceso de reescritura a su composición escrita (Scardamalia \& Bereiter, 1992). Por último, más allá de su potencial para el aprendizaje, la comunicación, la crítica y la expresión, la escritura cumple una función de evaluación y validación de los estudiantes (Navarro, 2018; Nesi \& Gardner, 2012), de sus conocimientos y capacidades, y también de sus posibilidades de graduación e ingreso a las culturas disciplinares y a los campos profesionales (Lillis, 2001). Sin embargo, este impacto de la escritura en las oportunidades de los estudiantes no suele corresponderse con suficiente tiempo y oportunidades de enseñanza y de aprendizaje de las formas de escritura solicitadas y esperadas.

De esta manera, existe una tensión entre la importancia y el impacto de la escritura en el sistema educativo y en las trayectorias estudiantiles, por un lado, y su invisibilidad en los planes de estudios, en los objetos de enseñanza y en las instrucciones y la retroalimentación docente, por el otro. En este contexto, los miembros de la comunidad educativa (funcionarios, docentes, estudiantes) se manejan con concepciones en general implícitas respecto del proceso de escritura, de las tareas de escritura solicitadas, de las formas de enseñanza y aprendizaje, de los criterios de evaluación o de la configuración de los géneros discursivos. Estas concepciones implícitas presentan un impacto significativo en los desempeños y las trayectorias estudiantiles (Villalón \& Mateos, 2009). Por este motivo, en los últimos años se han multiplicado las investigaciones que buscan comprender y contrastar las concepciones sobre la escritura de los estudiantes universitarios, vinculándolas con variables tales como desempeños letrados, autoeficacia, nivel educativo, disciplina de pertenencia y género, entre otras.

El presente artículo se inscribe en esta rica tradición de investigación, rastreando las teorías implícitas sobre la escritura académica y su enseñanza de estudiantes, en tres etapas diferenciadas de formación en educación superior: ingreso, transición y egreso. A partir de una encuesta respondida por 8182 estudiantes de una universidad metropolitana, estatal, tradicional y altamente selectiva de Chile, se reconstruyen las teorías implícitas sobre escritura académica y su enseñanza y se contrastan cuantitativamente, según etapa formativa, con prueba chi cuadrado de Pearson de bondad de ajuste.

\section{Teorías implícitas sobre escritura en educación superior}

La invisibilización de la escritura como objeto de enseñanza y aprendizaje se sustenta en un conjunto de creencias y concepciones frecuentes en los distintos miembros de la comunidad educativa, tanto estudiantes como docentes y funcionarios, y en los distintos niveles escolares. El concepto de teoría implícita, desarrollado para dar cuenta de teorías estructuradas sobre el aprendizaje, es especialmente operativo para abordar esta cuestión. Según Pozo, Scheuer, Mateos y del Puy Pérez Echeverría (2006), las teorías implícitas consisten en 'ciertos principios o supuestos básicos que, por su carácter implícito, suelen organizar nuestras acciones o decisiones sobre el aprendizaje y la enseñanza, y subyacer a ellas' (pp. 95-96). Estos principios teóricos, adquiridos por imitación o experiencia personal, constituyen un conocimiento procedimental y no declarativo que opera de forma rutinaria, automatizada y muchas veces eficiente y económica, para tomar decisiones en el aula; por eso, resultan muy difíciles de modificar. El concepto de teoría implícita permite colocar el foco en representaciones sociales vinculadas al ámbito de la enseñanza y el aprendizaje y en su carácter implícito, así como en su organización en un entramado teórico que impacta sobre las prácticas de los sujetos y que, por eso mismo, tiene efectos concretos y duraderos. A continuación, se propone un conjunto de teorías implícitas sobre la escritura y su enseñanza y 
aprendizaje, detallando su articulación y sus implicancias:

\section{1) La escritura es transcripción}

Según esta teoría implícita, la escritura no sería una herramienta epistémica que organiza y transforma las ideas y el pensamiento, sino que más bien funcionaría como una transcripción directa de una estructura conceptual previamente construida. A su vez, esta teoría implícita también significa que la escritura no media entre la realidad y su observación, sino que es una fotografía de los objetos y procesos existentes. Se vincula con la concepción general dominante sobre el lenguaje, denominada 'representacionalista' por Bronckart (2007): se borran los aspectos sociales, históricos y comunicativos del lenguaje para el entendimiento y la actividad humana, y se reemplaza por una visión determinista y biologicista. La escritura como trascripción se corresponde con una concepción transparente de la escritura, en la que esta no tiene ningún tipo de potencia transformadora o mediadora, y se opone a una concepción de la escritura como actividad retórica compleja (Russell, 2002) y al enfoque escribir para aprender (McCutchen, Teske, \& Bankston, 2008). Si se sostiene esta teoría implícita, entonces carece de sentido formar escritores que planifiquen y revisen sus textos, que tomen en cuenta las expectativas de los lectores o que construyan argumentos sólidos y convincentes según los marcos epistemológicos y las prácticas letradas de las distintas comunidades disciplinares.

\section{2) La escritura es un talento}

Para quien sostiene esta teoría implícita, la posibilidad de escribir de forma adecuada se distribuye de manera azarosa y desigual entre los distintos escritores, es decir, algunos escritores nacen con talento y otros no. Se basa en una concepción romántica de la autoría: el escritor no se expone a situaciones y textos recurrentes y tipificados que debe aprender, ni puede reconocer y adecuarse a las condiciones retóricas subyacentes utilizando técnicas y estrategias, sino que simplemente cuenta o no cuenta con la habilidad innata de escribir bien, y esa habilidad interna se activa en momentos de inspiración. Esta concepción invisibiliza las diferencias en el capital cultural y semiótico académico (Rose \& Martin, 2012), en las oportunidades de escribir géneros discursivos diversos y en las trayectorias escolares previas de los estudiantes. Además, al igual que la teoría de la escritura como transcripción, reduce las posibilidades de que las instituciones educativas enseñen a escribir.

\section{3) La escritura es inspiración individual}

Esta teoría implícita también corresponde a una concepción romántica de la escritura: el escritor inspirado escribiría su texto de forma casi inconsciente e instantánea como producto y sus decisiones escriturales responderían a motivaciones opacas e inexpugnables. A su vez, desde esta perspectiva la escritura es una empresa enteramente individual en la que no existe la negociación con otros lectores y escritores; el autor debe buscar espacios y momentos de aislamiento en donde fomentar su conexión única con la inspiración. De esta manera, se restringen las instancias de planificación y revisión de textos en situaciones de trabajo con pares o de supervisión por expertos, es decir, se trata de una concepción que desconoce la escritura como proceso complejo que puede ser modelado por tutores o construido y negociado de forma colectiva.

\section{4) La escritura es una habilidad básica}

Según esta teoría implícita, la escritura es una habilidad única, elemental y generalizable a diferentes contextos, que no reconoce niveles de dominio, roles de participación ni conjuntos de géneros discursivos, según el perfil del escritor. De esta manera, sería válido exigir a un estudiante ingresante a la universidad que escribiera un artículo científico, a pesar de que no se trata de un género de formación 
(Navarro, 2014), o sería esperable que toda la comunidad educativa coincidiera respecto de qué es un ensayo o un informe o qué se espera al solicitar 'comparar' o 'explicar'. Además, los diferentes entornos sociales no contarían con discursos y géneros discursivos característicos, vinculados a sus formas de conceptualizar el mundo, y las formas de escribir serían inmutables, sin modificaciones en el tiempo. Para quien sostiene esta concepción, la escritura es una habilidad que se adquiere en las etapas iniciales de la alfabetización, y por tanto la enseñanza de la escritura en la universidad tendría a lo sumo un objetivo remedial como ajuste de déficits formativos previos, y debería ubicarse curricularmente al comienzo de los estudios superiores (Russell, 2002).

\section{5) La escritura es vocabulario y ortografía}

En las ocasiones en que sí se considera que la escritura, en particular académica, constituye una herramienta que construye discursos específicos y que los estudiantes deben aprender a dominar, es frecuente que solo se atienda a algunos de sus rasgos más formales y visibles: la normativa lingüística (ortografía, puntuación, normas de cita) y, en menor medida, el vocabulario técnico disciplinar. Enseñar escritura se correspondería con transmitir reglas descontextualizadas o listas de términos. Quien sostiene esta teoría presta menor importancia a los aspectos vinculados a la construcción de textos cohesivos, con estructuras internas articuladas, y a la adecuación al contexto situacional y cultural, a las expectativas de los interlocutores y a los propósitos que el texto cumple en los diferentes sistemas de actividad (Bazerman, 2012).

\section{6) La escritura no se enseña}

Las teorías implícitas previas fomentan una desatención de la escritura como actividad retórica compleja que constituye un objeto válido y necesario de enseñanza y aprendizaje a través del currículum y de las etapas formativas, es decir, en las distintas asignaturas, y no solo en aquellas vinculadas al lenguaje, y en los distintos niveles educativos, no solo en educación básica (Bazerman et al., 2016). Tanto si se considera una habilidad básica funcional, una cuestión de talento innato e inspiración individual, una transcripción que reproduce cosas o pensamientos, o un conjunto de normas lingüísticas y un repertorio de vocabulario técnico, la escritura no debería ser enseñada, modelada ni retroalimentada, en particular en la universidad, que en cambio debería dedicarse a la enseñanza de contenidos disciplinares. Esta teoría implícita se vincula con la concepción del aprendizaje de la escritura por inmersión (es decir, aprender a escribir a través de la práctica sin enseñanza explícita, modelamiento ni retroalimentación), así como con la concepción de que la lectura de textos puede transferirse espontáneamente a la habilidad de escribirlos.

En suma, si un profesor sostiene algunas de estas teorías implícitas, entonces posiblemente no dedicará demasiado tiempo o esfuerzo a enseñar, fomentar o andamiar los procesos de escritura, no dará instrucciones pertinentes respecto de sus expectativas y sus formas de evaluación, ni incluirá la escritura en su programa de estudios o en los procesos de innovación curricular en los que participe. De manera similar, un estudiante que sostenga algunas de estas posiciones dedicará poco tiempo y esfuerzo a solicitar precisiones sobre lo que debe escribir, a buscar ejemplares aprobados de años previos para comprender las expectativas del profesor o a planificar y revisar su escrito antes de entregarlo. Por último, si un funcionario sostiene algunas de estas teorías implícitas, entonces es probable que considere que la enseñanza de la escritura en la universidad no es parte de un proceso necesario y válido de enculturación en prácticas letradas disciplinares nuevas, sino un problema que requiere ciertas acciones remediales y descontextualizadas para resolverse, es decir, el denominado mito de la transitoriedad (Russell, 2002). Así, las concepciones y percepciones de los miembros de la comunidad educativa sobre la escritura, su enseñanza y su aprendizaje 
impactan en sus acciones, en sus prácticas, en sus desempeños y en sus trayectorias (Pajares \& Valiante, 2006).

Esta centralidad de las teorías implícitas sobre la escritura en los desempeños letrados de los estudiantes y en las prácticas educativas de los profesores, ha multiplicado en los últimos años las investigaciones al respecto. Desde una perspectiva cuantitativa, White y Bruning (2005) revelan los contrastes entre los estudiantes que piensan que la escritura es fundamentalmente información que se reproduce y transmite desde fuentes autorizadas al lector (creencias transmisivas), quienes reflejan un compromiso cognitivo y afectivo limitado durante la escritura, y los estudiantes que creen que la escritura corresponde a un proceso más dialógico y constructivo (creencias transaccionales). Villalón y Mateos (2009) contrastan las concepciones de estudiantes de educación media y superior sobre las facetas de la escritura y hallan que estos últimos muestran una concepción más compleja y sofisticada de la escritura, si bien ninguno de los grupos alcanza plenamente una perspectiva epistémica. En un estudio similar, Castells, Mateos, Martín, Solé y Miras (2015) analizan la percepción sobre competencia en escritura académica en estudiantes de nueve universidades españolas y encuentran dos perfiles: estudiantes con una concepción más sofisticada de la escritura y, consecuentemente, más seguros sobre su competencia de escritura autopercibida, y estudiantes que otorgan menos importancia a la escritura y se muestran menos seguros sobre sus habilidades letradas. Más recientemente, Navarro, Uribe-Gajardo, Lovera-Falcón y Sologuren (en prensa) analizan cualitativamente las creencias y expectativas sobre escritura y su enseñanza en respuestas abiertas de 180 estudiantes ingresantes a la universidad en seis áreas de conocimiento; hallan que los estudiantes identifican dificultades en aspectos discursivos y procesuales de géneros poco precisos como el 'ensayo', y consideran que la escritura se aprende a partir de la práctica y la ejercitación activa, acompañada de enseñanza y retroalimentación de profesores y tutores, aunque desconocen los espacios institucionales y curriculares apropiados para aprender a hacerlo. Otras investigaciones, como Vázquez (2007), identifican las tareas o consignas de escritura como contrato que restringe las opciones del escritor y los resultados esperados, pero que muestra diferencias de interpretación tanto entre profesores y estudiantes como entre los propios estudiantes, ya que se produce una construcción de la tarea en la que intervienen expectativas personales y experiencias previas. En esta misma línea, Stagnaro y Chosco Díaz (2013) aportan evidencias cualitativas a partir del contraste de las representaciones sociales de estudiantes y profesores de una misma asignatura sobre tareas de escritura requeridas; hallan, por ejemplo, que para los profesores la tarea de relacionar implica vincular dos o más elementos (teorías, autores, fenómenos) de manera creativa en sus aspectos de convergencia y divergencia, pero que para la mayoría de los estudiantes solo se trata de relacionar los aspectos en común. Otras investigaciones abordan las concepciones sobre escritura, su aprendizaje y su enseñanza en instituciones de educación superior norteamericanas (Carlino, 2005); el impacto de las concepciones en el compromiso con la retroalimentación en las tareas de escritura (Han, 2017); la relación entre la percepción de autoeficacia, motivación y el desempeño escrito (SandersReio, Alexander, Reio, \& Newman, 2014); las metáforas que caracterizan las concepciones sobre escritura de los futuros profesores de lengua materna (Wittek, Askeland, \& Aamotsbakken, 2015); el contraste entre las representaciones sociales de profesores y estudiantes acerca de la escritura académica y de los géneros discursivos en cuatro comunidades disciplinares (Marinkovich, Velásquez, \& Córdova, 2012); y las concepciones de profesores sobre los géneros de formación estudiantil (Natale, 2011), sobre escritura en posgrado (Pereira \& Di Stefano, 2009), sobre elaboración de tesis (Tapia Ladino \& Marinkovich, 2011) y sobre diversidad e inclusión (Rivera Lam, 2017).

Como puede apreciarse, la bibliografía es diversa respecto del constructo teórico seleccionado (creencias, concepciones, 
expectativas, representaciones sociales, teorías implícitas), de la metodología seguida (análisis cuantitativo a partir de encuestas; análisis cualitativo a partir de grupos focales y entrevistas; análisis del discurso) y de las variables del grupo social estudiado (por disciplina, por nivel educativo, por región, por género, entre otras). Sin embargo, tiene en común la premisa de que las representaciones, concepciones y teorías implícitas varían según el perfil social del sujeto, en general permanecen implícitas, e impactan en sus desempeños letrados.

\section{Método}

Durante el segundo semestre de 2016, se elaboró un cuestionario compuesto de 16 preguntas cerradas y dos preguntas abiertas sobre lectura, escritura, su enseñanza y aprendizaje. El cuestionario fue validado por cuatro pares expertos en didáctica de la escritura académica, y posteriormente revisado por metodólogos y sociólogos del Departamento de Pregrado y de la Facultad de Ciencias Sociales de la universidad estudiada, a cargo de la elaboración de la encuesta de caracterización de la institución, de pretensión censal. Los estudiantes debieron leer y aceptar un consentimiento informado antes de acceder a las preguntas. El cuestionario fue de tipo autoaplicado en línea de respuesta voluntaria y se aplicó a través de la plataforma SurveyGizmo. Entre el 12/01/17 y el 09/03/17, se encuestó a los estudiantes ingresantes cohorte $2017(n=6309)$, con una tasa de respuesta del $81 \%$ ( $n$ : 5083); entre el 10/08/17 y el 11/09/17, se encuestó a los estudiantes de transición ( $n$ $=8664$ ), que ingresaron a la universidad en la cohorte 2014-2015, con una tasa de respuesta general del $22 \%(n=1884)$; y entre el $14 / 06 / 17$ y el 20/07/17 se encuestó a los graduados y titulados del año $2015(n=2881)$, con una tasa de respuesta del $42 \%(n=1215)$. En total, los datos provienen de 8182 estudiantes, casi la mitad de la población encuestada (46\%). En el presente artículo, se analizan las respuestas a las preguntas cerradas, que se detallan en la Figura 1.

\section{Figura 1}

Cuestionario sobre lectura y escritura y su enseñanza y aprendizaje en la universidad.

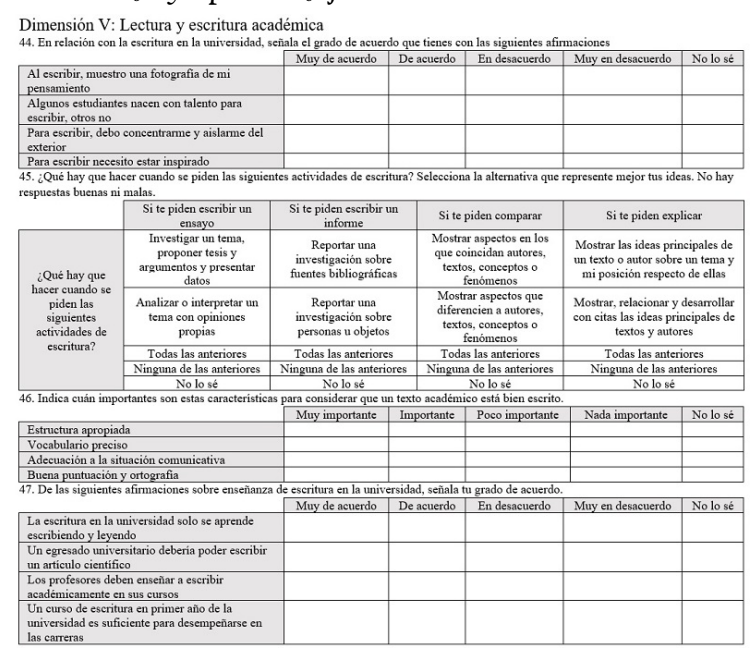

Las cuatro afirmaciones de la sección 44, presentadas con escala Likert, vinculan las teorías implícitas que consideran la escritura, respectivamente, como transcripción, como talento, como práctica individual y como inspiración. La sección 45 se especifica en una serie de géneros discursivos (ensayo e informe) y tareas de escritura (comparar y explicar) sobre las que no existe acuerdo generalizado, es decir, sobre las que los diferentes lectores y escritores de distintas áreas disciplinares y etapas formativas, muchas veces tienen expectativas implícitas diversas, como reportan estudios explorados más arriba. Se utilizaron intencionalmente denominaciones sin modificadores que pudieran brindar más información sobre el género discursivo, la tarea o la disciplina, de forma tal de fomentar la aparición de teorías implícitas diversas sobre los géneros y las tareas. En concreto, las opciones contrastan el rol que los estudiantes atribuyen a las opiniones propias, las fuentes bibliográficas y las coincidencias entre autores en la resolución de estas tareas y géneros. La sección 46, también con escala Likert, se vincula con distintas dimensiones articuladas que componen un texto académico; una teoría implícita que considera las dimensiones más formales y salientes de la escritura académica optará por brindar más 
peso a aspectos normativos y léxicos, mientras que una concepción de la escritura como actividad retórica compleja brindará más peso a los aspectos comunicativos y estructurales. La sección 47, también organizada en una escala Likert, ofrece un conjunto de afirmaciones vinculadas a las teorías implícitas que consideran que la escritura es una habilidad básica y que, por tanto, no se debe enseñar en la universidad.

Las respuestas del cuestionario se analizaron de forma descriptiva en relación con las teorías implícitas sobre escritura desarrolladas más arriba. Posteriormente, se buscó determinar si existían diferencias estadísticamente significativas entre los porcentajes de cada respuesta agrupada ( $\sin$ considerar las opciones 'no lo sé' ni 'ninguna de las anteriores') según cada cohorte, mediante la prueba de chi cuadrado de Pearson de bondad de ajuste, utilizando el software estadístico IBM SPSS Statistics 23. El nivel de confianza establecido fue del $95 \%$ (equivalente a un nivel de significación de $\left.{ }^{*} p<0.05\right)$.

\section{Resultados y discusión}

\section{Teoría implícita 1: La escritura es transcripción}

La medición de los grados de acuerdo respecto de la afirmación 'al escribir, muestro una fotografía de mi pensamiento' (véase 1.a en Tabla 1), de la sección 44 del cuestionario, muestra que ocho de cada 10 estudiantes sostienen la teoría implícita que considera la escritura como transcripción del pensamiento (media: 83.4 \%). Sin embargo, cuando los estudiantes ingresan efectivamente en la educación superior, se reduce el porcentaje que está de acuerdo con tal afirmación (ingreso: $88.1 \%$; transición: $73.6 \%$; egreso: 79.5 $\%)$, a la vez que aumenta el porcentaje de estudiantes que está en desacuerdo, con diferencias estadísticamente significativas entre todos los grupos; curiosamente, esta tendencia halla su porcentaje más alto en la transición, no en el egreso.

\section{Tabla 1}

Contraste de grados de acuerdo por etapa formativa con afirmaciones sobre escritura en la universidad

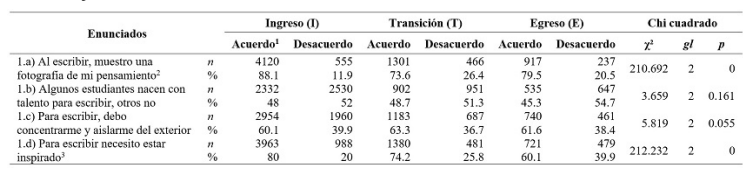

1 Acuerdo: agrupación de 'Muy de acuerdo' y 'De acuerdo'. Desacuerdo: agrupación de 'En desacuerdo' y 'Muy en desacuerdo'. 2 El cruce de los grupos de manera pareada fue significativo en todos los pares con $p$ $<0.001: \mathrm{I} / \mathrm{T} \chi^{2}=202.168 \mathrm{gl}=1$; I/E $\chi^{2}$ $=59.195 \mathrm{gl}=1$; T/E $\chi^{2}=13.006 \mathrm{gl}=1$.

3 El cruce de los grupos de manera pareada fue significativo en todos los pares con $p$ $<0.001: \mathrm{I} / \mathrm{T} \chi^{2}=21.749 \mathrm{gl}=1 ; \mathrm{I} / \mathrm{E} \chi^{2}=$ $211.905 \mathrm{gl}=1 ; \mathrm{T} / \mathrm{E} \chi^{2}=67.097 \mathrm{gl}=1$.

\section{Teoría implícita 2: La escritura es un talento}

Respecto de la afirmación 'algunos estudiantes nacen con talento para escribir, otros no' (véase 1.b en Tabla 1), que mide el grado de acuerdo con la teoría implícita de la escritura como talento innato, los estudiantes se distribuyen casi de forma equitativa entre aquellos que acuerdan (media: $47.7 \%$ ) y aquellos que la rechazan (media: $52.3 \%$ ). Esta teoría implícita se sostiene sin modificaciones significativas a lo largo de la educación superior.

Teoría implícita 3: La escritura es inspiración individual

Con la afirmación 'para escribir, debo concentrarme y aislarme del exterior' (véase 1.c en Tabla 1), se busca medir los grados de acuerdo con una teoría implícita de la escritura como práctica individual y aislada. Los resultados muestran que la mayoría de los estudiantes está de acuerdo o muy de acuerdo con esta posición (media: $61.1 \%$ ) y que la distribución se sostiene sin modificaciones significativas a lo largo de la educación superior.

A su vez, la mayoría de los estudiantes sostiene una teoría implícita de la escritura como inspiración, como muestra la medición de los grados de acuerdo con la afirmación 
'para escribir, necesito estar inspirado' (véase 1.d en Tabla 1), si bien la reducción en la proporción de estudiantes es marcada (ingreso: 80 \%; transición: $74.2 \%$; egreso: $60.1 \%$ ), estas diferencias son estadísticamente significativas.

\section{Teoría implícita 4: La escritura es una habilidad básica}

Para reconstruir las teorías implícitas de los estudiantes sobre los propósitos de géneros discursivos y tareas de escritura, se consultó a los estudiantes de las distintas cohortes sobre las denominaciones 'ensayo', 'informe', 'comparar' y 'explicar', frecuentes en educación superior, en la sección 45 del cuestionario. El propósito fue determinar si las concepciones y experiencias de los estudiantes, asociadas a estas denominaciones, se mantienen inalteradas a lo largo de la formación en educación superior, lo cual abonaría la existencia de una teoría implícita sobre la escritura como habilidad básica sin variaciones situadas.

\section{Tabla 2}

Contraste de concepciones sobre propósitos del género 'ensayo' por etapa formativa

\begin{tabular}{lrrrr}
\hline \multicolumn{1}{c}{ Enunciados } & Ingreso (I) & Transición (T) & Egreso (E) \\
\hline 2.a) Investigar un tema, proponer tesis y argumentos y presentar datos & $n$ & 1096 & 446 & 297 \\
2.b) Analizar o interpretar un tema con opiniones propias & $n$ & 21.8 & 24.3 & 25.1 \\
2.c) Todas las anteriores & $\%$ & 1268 & 712 & 513 \\
& $n$ & 25.3 & 38.8 & 43.4 \\
\hline
\end{tabular}

El cruce de los grupos de manera pareada fue significativo en todos los pares: $\mathrm{I} / \mathrm{T} \chi^{2}=$ $158.971 * * *, g l=2 ; \mathrm{I} / \mathrm{E} \chi^{2}=203.153 * * *, g l=2$; $\mathrm{T} / \mathrm{E} \chi^{2}=9.942 * *, g l=2$. El quitar la alternativa 2.a), y cruzar de manera pareada, fue significativo en todos los pares: I/T $\chi^{2}=156.672 * * *, g l=1$; $\mathrm{I} / \mathrm{E} \chi^{2}=202.628 * * *, g l=1 ; \mathrm{T} / \mathrm{E} \chi^{2}=9.708 * *$, $g l=1$. El quitar la alternativa 2.b), y cruzar de manera pareada, fue significativo sólo entre Ingreso y Transición e Ingreso y Egreso: $\mathrm{I} / \mathrm{T} \chi^{2}$ $=43.690 * * *, g l=1 ; \mathrm{I} / \mathrm{E} \chi^{2}=60.064 * * *, g l=$ $1 ; \mathrm{T} / \mathrm{E} \chi^{2}=3.739, g l=1, p=0.053$. El quitar la alternativa 2.c), y cruzar de manera pareada, fue significativo sólo entre Ingreso y Transición e Ingreso y Egreso: I/T $\chi^{2}=19.447 * * *, g l=$ $1 ; \mathrm{I} / \mathrm{E} \chi^{2}=23.028^{* * *}, g l=1 ; \mathrm{T} / \mathrm{E} \chi^{2}=0.693$, $g l=1, p=0.405$.***p $<0.001 \mathrm{y} * * p<0.05$

Respecto del género 'ensayo', las diferentes etapas formativas muestran un fuerte contraste respecto del propósito 'analizar o interpretar un tema con opiniones propias', como se detalla en la Tabla 2: mientras que la mayoría de los estudiantes aún sin experiencia en educación superior combina ese objetivo con otros en los que aparece menos la postura propia (52.9 $\%$ para ingreso), en las etapas subsiguientes se incrementa el rol del posicionamiento y la interpretación respecto de las temáticas abordadas como rasgo típico del género hasta resultar mayoritario entre las respuestas $(25.3 \%$ para ingreso, $38.8 \%$ para transición, $43.4 \%$ para graduación; con diferencias estadísticamente significativas entre los tres grupos).

Respecto del género 'informe', la Tabla 3 muestra que la mayoría de los estudiantes (en promedio $66.1 \%$ ) considera que el género reporta una investigación empírica que además incluye el uso de fuentes bibliográficas; además, se verifica una relativa estabilidad en esta concepción del género a lo largo de las diferentes etapas formativas. Sin embargo, cabe destacar una reducción en el porcentaje de estudiantes que considera que la investigación sobre fuentes bibliográficas forma parte del género (del $25.7 \%$ en el ingreso al $22.8 \%$ en el egreso).

\section{Tabla 3}

Contraste de concepciones sobre propósitos del género 'informe' por etapa formativa

\begin{tabular}{lrrrr}
\hline \multicolumn{1}{c}{ Enunciados } & Ingreso (I) & Transición (T) & Egreso (E) \\
\hline \multirow{2}{*}{ 3.a) Reportar una investigación sobre fuentes bibliográficas } & $n$ & 1235 & 396 & 252 \\
3.b) Reportar una investigación sobre personas u objetos & $\%$ & 25.7 & 22.5 & 22.8 \\
& $\%$ & 414 & 164 & 138 \\
3.c) Todas las anteriores & $n$ & 816 & 9.3 & 12.5 \\
& $\%$ & 65.7 & 1200 & 715 \\
\hline
\end{tabular}

El cruzar los grupos de manera pareada fue significativo en todos los pares: I/T $\chi^{2}=7.272 *, g l$ $=2 ; \mathrm{I} / \mathrm{E} \chi^{2}=17.545^{* *}, g l=2 ; \mathrm{T} / \mathrm{E} \chi^{2}=7.728^{*}$, $g l=2$. El quitar la alternativa 3.a), y cruzar de manera pareada, fue significativo sólo entre Ingreso y Egreso, y Transición y Egreso: I/T $\chi^{2}=0.171, g l$ $=1, p=0.679 ; \mathrm{I} / \mathrm{E} \chi^{2}=13.209 * *, g l=1$; T/E $\chi^{2}$ $=7.699^{*}, g l=1$. El quitar la alternativa 3.b), y cruzar de manera pareada, fue significativo sólo entre Ingreso y Transición: I/T $\chi^{2}=6.509 *, g l=1$; $\mathrm{I} / \mathrm{E} \chi^{2}=1.697, g l=1 ; p=0.193 ; \mathrm{T} / \mathrm{E} \chi^{2}=0.496$, $g l=1 p=0.481$. El quitar la alternativa 3.c), $\mathrm{y}$ cruzar de manera pareada, fue significativo sólo entre Ingreso y Egreso y Transición y Egreso: I/T $\chi^{2}=3.78, g l=1, p=0.052 ; \mathrm{I} / \mathrm{E} \chi^{2}=16.878 * *, g l=$ $1 ; \mathrm{T} / \mathrm{E} \chi^{2}=3.944^{*}, g l=1 .{ }^{*} p<0.05, * * p<0.01$

En el caso de la tarea de escritura 'comparar', el contraste más destacado entre las etapas de 
formación es que casi un tercio de los estudiantes ingresantes $(31.3 \%)$ considera que esta tarea solo focaliza en mostrar coincidencias, pero no diferencias y contrastes, como se ve en la Tabla 4. Esta concepción sobre el género se reduce notablemente ya en la etapa de transición (6.1 $\%)$, así como la concepción de que comparar solo consiste en mostrar similitudes (del $5.7 \%$ en ingreso a $1.9 \%$ en transición), y se mantiene estable hasta el egreso. Por el contrario, la mayoría de los estudiantes que ya ha atravesado procesos de enculturación en educación superior (transición: $92 \%$; egreso: $91.6 \%$ ) coincide en que la comparación involucra mostrar aspectos tanto comunes como contrastivos entre autores, textos, conceptos y fenómenos. Todas estas diferencias son estadísticamente significativas entre ingreso y cada uno de los otros grupos. Se verifica, por lo tanto, la transformación de una concepción de la tarea de escritura de los ingresantes que, luego, se mantiene estable en las etapas sucesivas de formación en educación superior.

\section{Tabla 4}

Contraste de concepciones sobre propósitos de la tarea 'comparar' por etapa formativa

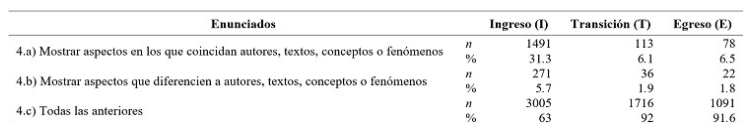

El cruzar los grupos de manera pareada fue significativo sólo entre Ingreso y Transición e Ingreso y Egreso: $\mathrm{I} / \mathrm{T} \chi^{2}=551.407 * *, g l=2$; $\mathrm{I} / \mathrm{E} \chi^{2}=362.921 * *, g l=2 ; \mathrm{T} / \mathrm{E} \chi^{2}=0.319, g l$ $=2, p=0.853$. El quitar la alternativa 4.a), y cruzar de manera pareada, fue significativo sólo entre Ingreso y Transición e Ingreso y Egreso: I/ $\mathrm{T} \chi^{2}=76.972 * *, g l=1 ; \mathrm{I} / \mathrm{E} \chi^{2}=52.852 * *, g l$ $=1 ; \mathrm{T} / \mathrm{E} \chi^{2}=0.21, g l=1 p=0.885$. El quitar la alternativa 4.b), y cruzar de manera pareada, fue significativo sólo entre Ingreso y Transición e Ingreso y Egreso: $\mathrm{I} / \mathrm{T} \chi^{2}=500.142 * *, g l=1 ; \mathrm{I} / \mathrm{E}$ $\chi^{2}=325.114^{* *}, g l=1 ; \mathrm{T} / \mathrm{E} \chi^{2}=0.292, g l=1$, $p=0.589$. El quitar la alternativa 4.c), y cruzar de manera pareada, fue significativo sólo entre Ingreso y Transición y Transición y Egreso: $\mathrm{I} / \mathrm{T} \chi^{2}$ $=7.856^{*}, g l=1 ; \mathrm{I} / \mathrm{E} \chi^{2}=3.127, g l=1, p=0.077$;

$\mathrm{T} / \mathrm{E} \chi^{2}=36.858^{* *}, g l=1 * p<0.05, * * p<0.001$

Por último, la tarea de escritura 'explicar' muestra, al igual que la tarea previa, un marcado contraste entre la cohorte de ingreso y las cohortes de estudiantes en etapas de transición y egreso de su formación universitaria, las cuales mantienen entre sí un grado relativamente alto de homogeneidad. Según la Tabla 5, el estudiante ingresante tiene ideas dispares sobre los propósitos de la tarea de 'explicar': aproximadamente, un tercio (39.4\%) considera que las citas bibliográficas ocupan un lugar central en el desarrollo y puesta en relación de las ideas principales de textos y autores, otra porción menor $(27.7 \%)$ opta por ponderar el rol de la posición personal sobre las ideas explicadas, mientras que otro tercio (33\%) cree que explicar involucra ambas cosas. En cambio, entre los estudiantes de transición y graduados, se incrementa el número que pondera el rol de las citas bibliográficas y la puesta en relación de textos y autores (transición: 53.9 \%; egreso: 53.3 $\%$; diferencias estadísticamente significativas entre ingreso y cada uno de los otros grupos), al mismo tiempo que se reduce la cantidad de estudiantes que piensa que explicar incluye expresar una posición propia (transición: 21 \%; egreso: $21.3 \%$; diferencias estadísticamente significativas entre ingreso y cada uno de los otros grupos). En conclusión, el proceso de enculturación en educación superior impacta en una concepción de la tarea de explicar menos vinculada a las posiciones personales y más centrada en el respaldo bibliográfico y la puesta en relación. 


\section{Tabla 5}

Contraste de concepciones sobre propósitos de la tarea 'explicar' por etapa formativa

\begin{tabular}{llrrr}
\hline \multicolumn{1}{c}{ Enunciados } & $\begin{array}{c}\text { Ingreso } \\
\text { (I) }\end{array}$ & $\begin{array}{c}\text { Transición } \\
\text { (T) }\end{array}$ & $\begin{array}{c}\text { Egreso } \\
\text { (E) }\end{array}$ \\
\hline 5.a). Mostrar las ideas principales de un texto o & $n$ & 1346 & 367 & 241 \\
autor sobre un tema y mi posición respecto de ellas & $\%$ & 27.7 & 21 & 21.7 \\
5.b). Mostrar, relacionar y desarrollar con citas las & $n$ & 1914 & 943 & 582 \\
ideas principales de textos y autores & $\%$ & 39.4 & 53.9 & 52.4 \\
5.c) Todas las anteriores & $n$ & 1603 & 439 & 288 \\
& $\%$ & 33 & 25.1 & 25.9 \\
\hline
\end{tabular}

El cruzar los grupos de manera pareada fue significativo sólo entre Ingreso y Transición e Ingreso y Egreso: $\mathrm{I} / \mathrm{T} \chi^{2}=111.103 * *, g l=2 ; \mathrm{I} /$ $\mathrm{E} \chi^{2}=63.095^{* *}, g l=2 ; \mathrm{T} / \mathrm{E} \chi^{2}=0.640, g l=2$, $p=0.726$. El quitar la alternativa 5.a), y cruzar de manera pareada, fue significativo sólo entre Ingreso y Transición e Ingreso y Egreso: $\mathrm{I} / \mathrm{T} \chi^{2}$ $=77.876^{* *}, g l=1 ; \mathrm{I} / \mathrm{E} \chi^{2}=44.260 * *, g l=1$; $\mathrm{T} / \mathrm{E} \chi^{2}=0.437, g l=1, p=0.509$. El quitar la alternativa 5.b), y cruzar de manera pareada, no fue significativo en ningún grupo: $\mathrm{I} / \mathrm{E} \chi^{2}=$ 0.003, $g l=1, p=0.956 ; \mathrm{I} / \mathrm{E} \chi^{2}=0.001, g l=$ $1, p=0.971 ; \mathrm{T} / \mathrm{E} \chi^{2}=0, g l=1, p=0.993$. El quitar la alternativa 5.c), y cruzar de manera pareada, fue significativo sólo entre Ingreso y

Transición e Ingreso y Egreso: $\mathrm{I} / \mathrm{T} \chi^{2}=70.256^{* *}$, $g l=1 ; \mathrm{I} / \mathrm{E} \chi^{2}=39.858 * *, g l=1 ; \mathrm{T} / \mathrm{E} \chi^{2}=$

0.399, $g l=1, p=0.528 . * p<0.05, * * p<0.001$

Teoría implícita 5: La escritura es vocabulario y ortografía

En la sección 46 del cuestionario se indagó en las teorías implícitas de los estudiantes sobre los niveles y componentes de la escritura y su importancia relativa. Para tal fin, se ofreció una escala Likert asociada a aspectos de la escritura, organizados desde lo más formal y descontextualizado a lo más general y situado, si bien en el cuestionario aparecen en un orden aleatorio: aspectos de normativa lingüística (puntuación y ortografía); uso de léxico técnico (vocabulario); organización y cohesión discursiva interna al texto (estructura); y propósitos, interlocutores, situación comunicativa y sistema de actividad (adecuación). La Tabla 6 resume los resultados.
Tabla 6

Contraste de grados de importancia por etapa formativa sobre características para considerar que un texto académico está bien escrito

\begin{tabular}{|c|c|c|c|c|c|c|c|c|c|}
\hline \multirow[b]{2}{*}{ anciac } & \multicolumn{2}{|c|}{ Ingreso (1) } & \multicolumn{2}{|c|}{ Transición (I) } & \multicolumn{2}{|c|}{ Egreso (E) } & \multicolumn{3}{|c|}{ Chi cuadrado } \\
\hline & Importante $^{2}$ & 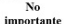 & Importante $^{1}$ & $\begin{array}{c}\text { No } \\
\text { Note }\end{array}$ & Importante & No & $x^{2}$ & $g l$ & $p$ \\
\hline 6.a) Istructura apropiada & 4988 & 82 & 1864 & 26 & 1194 & 11 & 3.479 & 2 & 0.178 \\
\hline & & $\begin{array}{l}1.6 \\
91\end{array}$ & & $\begin{array}{l}1.4 \\
17\end{array}$ & $\begin{array}{l}791 \\
1197\end{array}$ & 9 & 12.544 & 2 & 0.002 \\
\hline & $\begin{array}{l}98 . \\
492 \\
4\end{array}$ & 131 & & ${ }_{36}^{0.9}$ & 54 & ${ }_{33}^{0.7}$ & 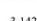 & , & \\
\hline & 97 & 2.6 & & 19 & & 2.8 & & $2^{2}$ & \\
\hline & 4981 & 93 & $\begin{array}{l}1858 \\
983 \\
983-20\end{array}$ & 33 & 989 & 13 & 3.360 & 2 & 0.18 \\
\hline
\end{tabular}

1 Importante: agrupación de 'Muy importante' e 'Importante'. No importante: agrupación de 'Poco importante' y 'Nada importante'. 2 El cruzar los grupos de manera pareada fue significativo solo entre Ingreso y Transición e Ingreso y Egreso: $\mathrm{I} / \mathrm{T} \chi^{2}=$ 7.24*, $g l=1 ; \mathrm{I} / \mathrm{E} \chi^{2}=6.826^{*}, g l=1 ; \mathrm{T} /$ $\mathrm{E} \chi^{2}=0.205, g l=1, p=0.651 . * p<0.05$

Prácticamente, todos los estudiantes, de todas las cohortes, otorgan grados de importancia similares a aspectos normativos (puntuación y ortografía; media: $98.3 \%$ ), léxicos (vocabulario preciso; media: $98.6 \%$ ), estructurales (estructura apropiada; media: $98.5 \%$ y situacionales (adecuación a la situación comunicativa; media: $97.5 \%$ ). Estos grados de acuerdo se mantienen constantes a lo largo de la formación universitaria, salvo en el caso del vocabulario técnico, que es considerado algo más importante a medida que los estudiantes avanzan en la enculturación académica (ingreso: $98.2 \%$; transición: $99.1 \%$; egreso: $99.3 \%$ ), con diferencias estadísticamente significativas. En suma, los estudiantes consideran que los aspectos más visibles, formales y normativos, enfatizados en las etapas formativas previas, tienen la misma importancia que los aspectos más discursivos, retóricos y situados, y la trayectoria universitaria no logra modificar esta concepción jerárquicamente indiferenciada de los aspectos que componen la escritura académica. De esta manera, los resultados sugieren que la enculturación disciplinar no logra formar escritores que prioricen ajustar más y mejor sus textos a diferentes situaciones, interlocutores y propósitos, o que jerarquicen su estructura y desarrollo temático y argumental, frente a otros aspectos más formales y menos situados. 


\section{Teoría implícita 6: La escritura no se enseña}

La medición del grado de coincidencia con la afirmación "la escritura en la universidad solo se aprende escribiendo y leyendo' (véase 7.a en Tabla 7) permite determinar la presencia de la teoría implícita respecto de que la escritura no requiere enseñanza explícita y curricularizada a lo largo de la educación superior y a través del currículum. En efecto, la mayoría de los estudiantes de todas las cohortes coincide con tal afirmación (ingreso: $66.5 \%$; transición: $72.3 \%$; egreso: $70.1 \%$ ), con un incremento estadísticamente significativo entre ingreso y las otras dos cohortes. Estos resultados sugieren que la mayoría de los estudiantes sostiene una teoría implícita de la escritura, reforzada en el trayecto por la universidad, como una práctica que se aprende a través de la ejercitación y, en consecuencia, sobre la que no sería necesaria (o quizás, a partir de sus experiencias, posible) la reflexión ni la enseñanza explícita.
Tabla 7

Contraste de grados de acuerdo por etapa formativa con afirmaciones sobre enseñanza de la escritura en la universidad

\begin{tabular}{|c|c|c|c|c|c|c|c|c|c|}
\hline \multirow{2}{*}{ Enunciados } & \multicolumn{3}{|c|}{ Ingreso (I) } & \multicolumn{2}{|c|}{ Transición (I) } & \multicolumn{2}{|c|}{ Egreso (E) } & \multicolumn{2}{|c|}{ Chi cuadrado } \\
\hline & & Acuerdo ${ }^{\prime}$ & Desacuerdo & Acuerdo & Desacuerdo & Acuerdo & Desacuerdo & $x^{2}$ & $g^{l}$ \\
\hline \multirow{2}{*}{$\begin{array}{l}\text { 7.a) La escritura en la univeresidad sólo } \\
\text { se apronde oscribiendo y y loyendoto }\end{array}$} & $n$ & 3240 & 1630 & 1345 & 515 & 840 & 358 & \multirow{2}{*}{$22.691 \pm *$} & \multirow[t]{2}{*}{2} \\
\hline & $\%$ & 66.5 & 33.5 & 72.3 & 27.7 & 70.1 & 29.9 & & \\
\hline \multirow{2}{*}{ 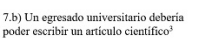 } & $n$ & 4222 & 661 & 1617 & 247 & 1000 & 201 & \multirow{2}{*}{$9.217^{*}$} & 2 \\
\hline & $\%$ & 86.5 & 13.5 & 86.7 & 13.3 & 83.3 & 16.7 & & \\
\hline \multirow{2}{*}{ 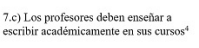 } & $n$ & 4443 & 466 & 1609 & 248 & 1031 & 170 & \multirow[t]{2}{*}{$33.788^{* *}$} & 2 \\
\hline & $\%$ & 90.5 & 9.5 & 86.6 & 13.4 & 85.8 & 14.2 & & \\
\hline \multirow{2}{*}{ 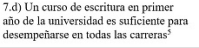 } & $n$ & 2004 & 2548 & $62 !$ & 1141 & 344 & 813 & \multirow{2}{*}{$98.159 \%$} & 2 \\
\hline & $\%$ & 44 & 56 & 35.2 & 64.8 & 29.7 & 70.3 & & \\
\hline
\end{tabular}

1 Acuerdo: agrupación de 'Muy de acuerdo' y 'De acuerdo'. Desacuerdo: agrupación de 'En desacuerdo' y 'Muy en desacuerdo'. $2 \mathrm{El}$ cruzar los grupos de manera pareada fue significativo solo entre Ingreso y Transición e Ingreso y Egreso: $\mathrm{I} / \mathrm{T} \chi^{2}=20.723 * *, g l=1 ; \mathrm{I} /$ $\mathrm{E} \chi^{2}=5.616^{*}, g l=1 ; \mathrm{T} / \mathrm{E} \chi^{2}=1.721, g l=1$.

$3 \mathrm{El}$ cruzar los grupos de manera pareada fue significativo solo entre Ingreso y Egreso y Transición y Egreso: $\mathrm{I} / \mathrm{T} \chi^{2}$ $=0.95, g l=1, p=0.758 ; \mathrm{I} / \mathrm{E} \chi^{2}=$ $8.113^{*}, g l=1$; T/E $\chi^{2}=7.108^{*}, g l=1$.

4 El cruzar los grupos de manera pareada fue significativo solo entre Ingreso y Transición e Ingreso y Egreso: $\mathrm{I} / \mathrm{T} \chi^{2}=$ $21.291 * *, g l=1 ; \mathrm{I} / \mathrm{E} \chi^{2}=22.489 * *, g l$ $=1 ; \mathrm{T} / \mathrm{E} \chi^{2}=0.396, g l=1, p=0.529$. $5 \mathrm{El}$ cruzar los grupos de manera pareada fue significativo en todos los pares: $\mathrm{I} / \mathrm{T} \chi^{2}=40.320^{* *}$, $g l=1 ; \mathrm{I} / \mathrm{E} \chi^{2}=77.83^{* *}, g l=1 ; \mathrm{T} / \mathrm{E} \chi^{2}=$ 9.588*, $g l=1 . * p<0.05, * * p<0.001$

Respecto de la afirmación 'los profesores deben enseñar a escribir académicamente en sus cursos' (véase 7.c en Tabla 7), que da cuenta de una teoría implícita de enseñanza de la escritura académica a través del currículum, los resultados muestran que la inmensa mayoría de los estudiantes está de acuerdo con ella (ingreso: $90.5 \%$; transición: $86.6 \%$; egreso: 85.8 $\%)$. Sin embargo, el ingreso a la universidad reduce el porcentaje de estudiantes que adopta esta perspectiva, es decir, menos estudiantes consideran que la escritura debería ser un objeto de enseñanza explícito de todas las carreras y asignaturas; este contraste es estadísticamente significativo.

La pregunta por el grado de acuerdo con 'un curso de escritura en primer año de la universidad es suficiente para desempeñarse en todas las carreras' (véase 7.d en Tabla 7) está sostenida por una teoría implícita de la escritura 
como habilidad básica (véase subsección previa) y el mito de la transitoriedad, es decir, la escritura como problema solucionable con un curso específico, no como práctica que debe enseñarse y ejercitarse a lo largo del currículum y de los niveles formativos. Las respuestas a esta consulta muestran que alrededor de uno de cada tres estudiantes está de acuerdo o muy de acuerdo con una posición afin al mito de la transitoriedad (media: $39.7 \%$ ), si bien el porcentaje de estudiantes se reduce con claridad a medida que se avanza en los estudios superiores (ingreso: $44 \%$; transición: $35.2 \%$; egreso: $29.7 \%$ ); estas diferencias son estadísticamente significativas.

Finalmente, la medición de los grados de acuerdo con la afirmación 'un egresado universitario debería poder escribir un artículo científico' (véase 7.b en Tabla 7) busca identificar las concepciones de los estudiantes respecto de las prácticas letradas al egreso de la educación superior: como conjunto de prácticas generales, comunes a todos los escritores (véase teoría implícita anterior), o como prácticas situadas específicas que las carreras deben incluir en su perfil de egreso. Los resultados indican que la inmensa mayoría de los estudiantes de todas las etapas formativas (ingreso: $86.5 \%$; transición: $86.7 \%$; egreso: $83.3 \%$ ) considera que el perfil de egreso debería incluir la capacidad de escritura de artículos científicos. Este hallazgo es sorprendente porque la escritura científica no es necesariamente una habilidad propia del perfil profesional en todas las carreras universitarias, ni tampoco una práctica generalizada en la inserción profesional de los graduados. A pesar de esta tendencia general, la cohorte de egreso muestra un menor grado de acuerdo con esta afirmación (diferencias estadísticamente significativas): el estudiante graduado parece más interiorizado con la diversidad de prácticas letradas, no necesaria ni exclusivamente científicas, que implica el tránsito por la educación superior.

En suma, las respuestas al cuestionario muestran una aparente contradicción: la mayoría de los estudiantes cree que la escritura solo se aprende a partir de la práctica, pero también coinciden en que todas las carreras y asignaturas deberían enseñar explícitamente a escribir, incluso artículos científicos, y que un curso de escritura de primer año es insuficiente para lograrlo. A medida que los estudiantes avanzan en su formación universitaria, estas posiciones se acomodan: menos estudiantes creen que se debería enseñar explícitamente y más estudiantes consideran que se aprende solo practicando, que no les alcanzaría un curso a comienzos de la carrera y que un graduado no necesariamente puede escribir artículos científicos. Estos resultados son preocupantes porque sugieren que la experiencia universitaria muestra posibilidades reducidas de enseñanza intencionada y andamiada de la escritura y, por tanto, los graduados ofrecen una concepción más escéptica sobre cómo se enseña y aprende en educación superior.

\section{Conclusiones y proyecciones futuras}

Esta investigación ha propuesto, relevado y contrastado un conjunto de teorías implícitas estudiantiles sobre la escritura y su enseñanza y aprendizaje en tres etapas diferenciadas de la formación en educación superior (ingreso, transición y egreso), a partir de las respuestas de un número elevado y representativo de estudiantes (8182 encuestados, $46 \%$ de la población). El objetivo ha sido identificar el grado de estabilidad o transformación de estas concepciones a medida que los estudiantes avanzan en sus trayectorias de formación como escritores disciplinares. Las teorías implícitas abordadas (escritura como transcripción, talento, inspiración individual, habilidad básica, vocabulario y ortografía, y objeto no enseñable) fomentan en la comunidad educativa una invisibilización de la escritura académica como práctica retórica compleja, ubicua, disciplinarmente específica, socialmente construida y negociada, organizada en tareas de escritura y géneros discursivos diversos, y objeto explícito y curricularizado de enseñanza y aprendizaje a través del currículum y de la formación en educación superior. 
El panorama reconstruido de teorías implícitas muestra una situación compleja y paradójica: algunas de estas teorías implícitas sobre escritura predominan entre los estudiantes (escritura como ortografía y transcripción del pensamiento), mientras que otras son mayoritariamente rechazadas (escritura como habilidad básica), y en un caso excepcional aparece una distribución equitativa entre quienes la rechazan y sostienen (escritura como talento innato). Al mismo tiempo, algunas de estas teorías prácticamente no se modifican a lo largo del proceso de enculturación en educación superior (importancia relativa de la adecuación situacional y la estructura textual frente a aspectos más formales y normativos), mientras que otras muestran una modificación significativa (escritura como inspiración y transcripción del pensamiento, importancia del vocabulario técnico). La escritura se entiende como objeto de enseñanza de todas las carreras y asignaturas, pero al mismo tiempo como aprendizaje que solo se produce mediante la práctica, si bien la experiencia universitaria ajusta posiciones: menos enseñanza docente explícita y más responsabilidad por el aprendizaje en la práctica estudiantil. Por su parte, las concepciones sobre géneros discursivos y tareas de escritura muestran en general que ciertos rasgos no se modifican demasiado al atravesar la educación superior (investigar empíricamente con fuentes bibliográficas en informes), mientras que otros se instalan a partir de la enculturación disciplinar (posicionamiento propio en el ensayo, exploración de similitudes y diferencias en comparaciones, uso de citas en explicaciones). En suma, los estudiantes ingresan con algunas teorías implícitas que pueden obstaculizar su proceso de enculturación en las prácticas letradas de las disciplinas, pero también con otras que lo favorecen; al mismo tiempo, la educación superior transforma parcialmente algunas de esas teorías implícitas, pero deja intactas otras.

Reconocer en qué medida los estudiantes sostienen estas teorías implícitas no solo hace aportes al conocimiento disciplinar, sino que también informa las iniciativas pedagógicas y las políticas institucionales de alfabetización académica y apoyo a los aprendizajes que necesariamente trabajan con concepciones y creencias de los estudiantes, y que pueden aprovechar qué aspectos enfatizar para lograr mejores resultados en sus programas y cursos. En investigaciones futuras, se deberá evaluar la posibilidad de generalizar estos resultados a otras instituciones y países, así como contrastar la distribución de las teorías implícitas hacia dentro de las áreas disciplinares y entre otros miembros de la comunidad educativa.

\section{Agradecimientos}

Se agradece el financiamiento otorgado por el Fondo Basal para Centros de Excelencia proyecto FB0003 de PIA-CONICYT, por el Proyecto FONDECYT № 1191069 de CONICYT y por el Plan de Mejoramiento Institucional (PMI) UCH1501 del Ministerio de Educación, Chile. También se agradece la colaboración de la Facultad de Ciencias Sociales de la universidad investigada en la construcción de la encuesta, de los cuatro expertos consultados en la revisión del instrumento de recolección y del Área de Gestión Académica de la universidad investigada en la recolección de los datos. Por último, se agradecen las valiosas sugerencias de Gabriela Gómez Vera durante la elaboración de este escrito.

\section{Referencias}

Bazerman, C. (2012). Actos de habla, géneros y sistemas de actividades: de qué manera los textos organizan las actividades y los grupos sociales. En Géneros textuales, tipificación y actividad (pp. 122-161). Puebla, MX: Benemérita Universidad Autónoma de Puebla.

Bazerman, C. (2013). Understanding the lifelong journey of writing development. Infancia $y$ Aprendizaje, 36(4), 421-441. https://doi.org /10.1174/021037013808200320

Bazerman, C., Little, J., Bethel, L., Chavkin, T., Fouquette, D., \& Garufis, J. (2016). Escribir a través del Currículum. Una guía 
de referencia. Córdoba, AR: Universidad Nacional de Córdoba.

Bronckart, J. -P. (2007). La enseñanza de lenguas: para una construcción de las capacidades textuales. En J. -P. Bronckart (Ed.), Desarrollo del lenguaje y didáctica de las lenguas (pp. 133-146). Buenos Aires: Miño y Dávila. Recuperado de https://archive-ou verte.unige.ch/unige:86402

Carlino, P. (2005). Representaciones sobre la escritura y formas de enseñarla en universidades de América del Norte. Revista de Educación, 336, 143-168. Recuperado de https://www.aacademica.org/paula.carli no/130.pdf

Carlino, P. (2013). Alfabetización académica diez años después. Revista Mexicana de Investigación Educativa, 18(57), 355-381. Recuperado de http://www.redalyc.org/arti culo.oa?id $=14025774003$

Castells, N., Mateos, M., Martín, E., Solé, I., \& Miras, M. (2015). Profiles of self-perceived competencies and conceptions of academic writing in university students. Cultura $y$ Educación, 27(3), 569-593. https://doi.org/ 10.1080/11356405.2015.1072358

Fernández Lamarra, N., \& Costa de Paula, M. F. (2011). [Introducción]. En N. Fernández Lamarra \& M. F. Costa de Paula (Comps.), La democratización de la educación superior en América Latina. Límites y posibilidades (pp. 9-34). Buenos Aires: Editorial de la Universidad Nacional de Tres de Febrero.

Han, Y. (2017). Mediating and being mediated: Learner beliefs and learner engagement with written corrective feedback. System, 69, 133-142. https://doi.org/10.1016/j.syste m.2017.07.003

Lillis, T. (2001). Student writing. Access, regulation, desire. Nueva York: Routledge.

Marinkovich, J., Velásquez Rivera, M., \& Córdova Jiménez, A. (Eds.). (2012). Comunidades académicas y culturas escritas: construcciones discursivas desde las ciencias y las humanidades. Valparaíso, CL: Ediciones Universitarias de Valparaíso.

McCutchen, D., Teske, P., \& Bankston, C. (2008). Writing and cognition: Implications of the cognitive architecture for learning to write and writing to learn. En C. Bazerman (Ed.), Handbook of research on writing: History, society, school, individual, text (pp. 554-578). Nueva York: Taylor \& Francis.

Natale, L. (2011). La enseñanza y el aprendizaje de los géneros discursivos en la educación superior. Interacciones entre las representaciones de los profesores universitarios sobre la escritura académica, las valoraciones acerca de los estudiantes y la elección de los géneros solicitados en las materias. Texturas, $10(10-11)$, 87-109. https:// www.academia.edu/5021884/La_ense\%C3\% B1anzay_el_aprendizaje_de_los_g\%C3\%A9ner os_discursivos_en_el_nivel_superior._An\%C3 \%Allisis_de_representaciones_en_el_discurso _de_docentes_universitarios

Navarro, F. (2014). Géneros discursivos e ingreso a las culturas disciplinares. Aportes para una didáctica de la lectura y la escritura en educación superior. En F. Navarro (Ed.), Manual de escritura para carreras de humanidades (pp. 29-52). Buenos Aires: Universidad de Buenos Aires.

Navarro, F. (2018). Más allá de la alfabetización académica: las funciones de la escritura en educación superior. En M. A. Alves \& V. Iensen Bortoluzzi (Eds.), Formação de Professores: Ensino, linguagens e tecnologias (pp. 13-49). Porto Alegre: Editora Fi.

Navarro, F., Uribe Gajardo, F., Lovera Falcón, P. $\&$ Sologuren, E. (en prensa). Encuentros con la escritura en el ingreso a la educación superior: representaciones sociales de estudiantes en seis áreas de conocimiento. Ibérica, 38 (otoño 2019).

Nesi, H., \& Gardner, S. (2012). Genres across the disciplines. Student writing in higher education. Cambridge: Cambridge University Press.

Pajares, F., \& Valiante, G. (2006). Selfefficacy beliefs and motivation in writing development. En C. A. MacArthur, S. Graham \& J. Fitzgerald (Eds.), Handbook of writing research (pp. 158-170). Nueva York: Guilford. 
Pereira, C., \& di Stefano, M. (2009). Representaciones acerca de la tesis doctoral en las reescrituras de la introducción. Un estudio de caso. En E. Arnoux (Ed.), Escritura y producción de conocimiento en las carreras de posgrado (pp. 209-226). Buenos Aires: Santiago Arcos Editor.

Pozo, J. I., Scheuer, N., Mateos, M., \& del Puy Pérez Echeverría, M. (2006). Las teorías implícitas sobre el aprendizaje y la enseñanza. En J. I. Pozo et al. (Eds.), Nuevas formas de pensar la enseñanza y el aprendizaje (pp. 95-132). Barcelona: Graó.

Prior, P., \& Bilbro, R. (2011). Academic enculturation: Developing literate practices and disciplinary identities. En M. Castelló (Ed.), University writing: Selves and texts in academic societies (pp. 19-31). Londres: Emerald Group Publishing.

Rivera Lam, M. (Ed.). (2017). Antropología de la escritura: representaciones de la diversidad y la inclusión en la Universidad de Antofagasta. Antofagasta, CL: Universidad de Antofagasta.

Rose, D., \& Martin, J. R. (2012). Learning to write. Reading to learn. Genre, knowledge and pedagogy in the Sydney School. Londres: Equinox Publishing.

Russell, D. (2002). Writing in the academic disciplines: A curricular history. Carbondale, IL: Southern Illinois University Press.

Sanders-Reio, J., Alexander, P. A., Reio, T. G. J., $\&$ Newman, I. (2014). Do students' beliefs about writing relate to their writing selfefficacy, apprehension, and performance? Learning and Instruction, 33, 1-11. https://d oi.org/10.1016/j.learninstruc.2014.02.001

Scardamalia, M., \& Bereiter, C. (1992). Dos modelos explicativos de los procesos de composición escrita. Infancia y Aprendizaje, 58, 43-64. Recuperado de https://dialnet.u nirioja.es/servlet/articulo?codigo $=48395$

Schleppegrell, M. J. (2004). The language of schooling. Mahwah, NJ: Erlbaum.

Stagnaro, D., \& Chosco Díaz, C. (2013).
Discordancias entre expectativas docentes y representaciones de los estudiantes en torno a las tareas de escritura académica: diagnóstico y algunas propuestas de intervención. En L. Natale (Ed.), El semillero de la escritura. Las tareas escritas a lo largo de tres carreras de la UNGS (pp. 95-128). Buenos Aires: Universidad Nacional de General Sarmiento.

Starke-Meyerring, D., \& Paré, A. (2011). The roles of writing in knowledge societies: Questions, exigencies, and implications for the study and teaching of writing. En D. Starke-Meyerring, A. Paré, N. Artemeva, M. Horne \& L. Yousoubova (Eds.), Writing in knowledge societies (pp. 3-28). Fort Collins, CO: The WAC Clearinghouse \& Parlor Press.

Swales, J. M. (2004). Research genres. Exploration and applications. Glasgow, UK: Cambridge University Press.

Tapia Ladino, M., \& Marinkovich, J. (2011). Representaciones sociales sobre la escritura de la tesis en la formación académica inicial en el área de las ciencias del mar. Onomázein, 24(2), 273-297. Recuperado de https://www.redalyc.org/articulo.oa?id= 134522498012

Vázquez, A. (2007). Consignas de escritura: entre la palabra del docente y los significados de los estudiantes. Colección de Cuadernillos de Actualización para Pensar la Enseñanza Universitaria, 2(7), 4-16. Recuperado de https://www.unrc.edu.ar/unrc/academic a/docs/publicaciones/vazquez-set07.pdf

Villalón, R., \& Mateos, M. (2009). Concepciones del alumnado en secundaria y universidad sobre la escritura académica. Infancia y Aprendizaje, 32 (2), 219-232. Recuperado de Recuperado de https://dialnet.unirioja.es/se rvlet/articulo? codigo $=2967269$

White, M., \& Bruning, R. (2005). Implicit writing beliefs and their relation to writing quality. Contemporary Educational Psychology, 30, 166-189. https://doi.org/10. 1016/j.cedpsych.2004.07.002 
Wittek, A. L., Askeland, N., \& Aamotsbakken,

B. (2015). Learning from and about writing:

A case study of the learning trajectories of student teachers. Learning, Culture and

Social Interaction, 6, 16-28. https://doi.org/1

0.13140/RG.2.1.1992.3364

\section{Notas}

* Artículo de investigación. 